\title{
INDONESIAN ARMY TERRITORIAL COMMANDERS 1950-MARCH 1983*
}

\author{
Compiled by the Editors
}

In July 1950, seven months after the transfer of sovereignty the Indonesian Army Chief of Staff (KSAD) Col. A. H. Nasution, issued an order dividing Indonesia into seven military territories (Territorium dan Tentara, $T \& T$ ), each under a divisional command. ${ }^{1}$ Two were on Sumatra, three on Java, the sixth was for Kalimantan, and the last covered the "Great East."

$\begin{array}{ll}\text { Command } & \text { Division Name } \\ \text { T \& T I } & \text { Bukit Barisan } \\ \text { T \& T II } & \text { Sriwijaya } \\ \text { T \& T III } & \text { Siliwangi } \\ \text { T \& T IV } & \text { Diponegoro } \\ \text { T \& T V } & \text { Brawijaya } \\ \text { T \& T VI } & \text { Tanjungpura } \\ \text { T \& T VII } & \text { Wirabuana }\end{array}$

Territory

Aceh, North \& West Sumatra, Riau

South Sumatra, Jambi

West Java

Central Java

East Java

Kalimantan

Eastern Indonesia (including

Sulawesi, Maluku, Bali, \& the

Lesser Sundas)

\author{
$H Q$ \\ Medan \\ Palembang \\ Bandung \\ Semarang \\ Malang \\ Banjarmasin \\ Makassar
}

These military territories were unrealistically large and corresponded to no historical or natural territorial divisions; nor did they approximate the local boundaries between military commands during the Revolution. From 1955 on, they underwent major reorganization, eventuating in seventeen Regional Military Commands (Komando Daerah Militer, KDM, later Kodam) by 1962 , a number that was reduced to sixteen when Kodam XI (Central Kalimantan) was abolished in 1974. Most of these changes were initiated in response to the regional crises of the mid-1950s, and were part of an effort by the army high command to institutionalize its authority and increase its control over the regional military leaders. These changes will be noted under the individual territorial listings.

The basic pattern followed in the listing is that of the "Current Data on Indonesia's Military Elite," periodically appearing in Indonesia. Less information, however, is given on the men holding the post of panglima than is usually provided on the members of the military elite. Where it is available, only the following data is given for each position: the name of the occupant, the date of his assumption of the office, the length of his term, his divisional or service background, and his ethnic origin. (For those early officeholders who had no previous divisional affiliation, we have listed the region where they were active during the Revolution.)

\footnotetext{
* So far as we can ascertain this list is accurate up to the March 1983 MPR session
} at which Soeharto was elected to a fourth Presidential term.

1. Keputusan KSAD No. 83/KSAD/Pnt/50, tt. July 20, 1950. 


\begin{tabular}{ccccc}
\multicolumn{4}{c}{ Sample } \\
Date of & Number of Months & Name of & Divisional & Ethnic \\
Installation & in Office & Occupant & Background & Origin
\end{tabular}

If a commander has held the post of panglima on more than one occasion, a single asterisk marks his first assignment, two asterisks his second, and so on. An alphabetical listing of the panglima, indicating the commands they held, is appended as a reference.

$$
\begin{aligned}
& \mathrm{B}=\text { B rawijaya } \\
& \mathrm{BB}=\text { Bukit Barisan } \\
& \mathrm{CPM}=\text { Corps Polisi Militer } \\
& \mathrm{D}=\text { Diponegoro }
\end{aligned}
$$

\author{
Abbreviations \\ $\mathrm{J} \quad=$ Javanese \\ RPKAD = Resimen Para Komando Angkatan Darat \\ $\mathrm{S} \quad=$ Sundanese \\ S1 = Siliwangi
}

\section{SUMATRA \\ $T \& T I$}

The territorial command in northern Sumatra was established on November 1 , 1950. It was officially designated $T \& T$ I Bukit Barisan (Aceh, North, and Central Sumatra [including West Sumatra and Riau]) on June 21, 1951.
[28.xii. $49 \quad 3 \frac{1}{2}$
*Col. Alex Evart Kawilarang
Siliwangi
Menadonese]
19.iv. $50 \quad 80$
**Col. Maludin Simbolon
$\mathrm{N} \& \mathrm{~S}$ Sumatra
Toba Batak
$27 . x i 1.56$
*Col. Djamin Gintings
$\mathrm{N}$ Sumatra
Karo Batak

In December 1956 dissident military officers took the first steps that led to the 1958 PRRI/Permesta rebellions against the central government. A "Banteng Council" seized control in West Sumatra on December 22 and Col. Simbolon formed a military junta two days later to seize power in North Sumatra. His Chief of Staff, Col. Djamin Gintings, with backing from the army high command, ousted him on December 27 , but was unable to assert himself in his predecessor's office. Within three months, T \& T I had effectively collapsed into three independent regions: Lt. Col. Ahmad Husein headed the Banteng Council in West Sumatra, which claimed authority over the whole of Central Sumatra; Major Sjamaun Gaharu established a KDM in Aceh; and Col. Djamin Gintings was panglima of the remnants of the Bukit Barisan command in North Sumatra. The autonomous territories in Central Sumatra and Aceh were reluctantly recognized by the central government on March 31, 1957 . When the full-scale rebellion broke out nearly a year later, the Army Chief of Staff responded by issuing a decree freezing the military command in Central Sumatra on February 12, 1958, and sending in an expeditionary force to suppress the Banteng Council. This meant establishment of the August 17th Operational Command in March 1958, which was transformed into a Komando Daerah Militer (III, 17 Agustus) in April 1959. The territories of $\mathrm{T} \& \mathrm{~T} \mathrm{I}$ in Aceh, North, and West Sumatra were then officially designated Kodam I, Kodam II, and Kodam III. 
Kodam I

Kodam I Iskandarmuda (Aceh)

\begin{tabular}{|c|c|c|c|c|}
\hline 30.iii. 57 & 43 & Lt. Col. Sjamaun Gaharu & Aceh & Acehnese \\
\hline $8 . x i .60$ & 34 & *Col. Moch. Jasin & B & $\mathbf{J}$ \\
\hline$? . \mathrm{ix} .63$ & 12 & Col. Nja' Adam Kamil & Aceh & Acehnese \\
\hline $21 . \mathrm{ix} .64$ & 33 & $\begin{array}{l}\text { *Brig. Gen. Moch. Ishak } \\
\text { Djuarsa }\end{array}$ & $\mathrm{Sl}$ & $\mathbf{S}$ \\
\hline 12. vi. 67 & 40 & Brig. Gen. Teuku Hamzah & N Sumatra & Acehnese \\
\hline $14 . x \cdot 70$ & 36 & $\begin{array}{l}\text { *Brig. Gen. Aang Kunaefi } \\
\text { Kartawirja }\end{array}$ & $\mathrm{S} 1$ & $\mathbf{S}$ \\
\hline $8 . x .73$ & 51 & Brig. Gen. A. Rivai Harahap & BB & $\begin{array}{l}\text { Mandailing } \\
\text { Batak }\end{array}$ \\
\hline $4 . \mathrm{i} .78$ & $48 \frac{1}{2}$ & Brig. Gen. R. A. Saleh & Sl & $\mathrm{S}$ \\
\hline $18 . \mathrm{i} .82$ & & Maj. Gen. Djoni Abdulrachman & $?$ & $?$ \\
\hline
\end{tabular}

\section{Kodam II}

Kodam II Bukit Barisan (North Sumatra)

\begin{tabular}{|c|c|c|c|c|}
\hline & 48 & **Col. Djamin Gintings & $\mathrm{N}$ Sumatra & Karo Batak \\
\hline $4 . i .61$ & 30 & Lt. Col. A. Manaf Lubis & N Sumatra & $\begin{array}{l}\text { Mandailing } \\
\text { Batak }\end{array}$ \\
\hline 3. vii. 63 & 1 & Col. A. Thalib & N Sumatra & Minangkabau \\
\hline 1. viii. 63 & 27 & Brig. Gen. Darjatmo & $\mathrm{D}$ & $J$ \\
\hline $29 . \times .65$ & 20 & $* *$ Brig. Gen. P. Sobiran & $\mathrm{B}$ ? & $?$ \\
\hline 25. vi. 67 & 12 & $\begin{array}{l}\text { *Brig. Gen. Sarwo Edhie } \\
\text { Wibowo }\end{array}$ & $\mathrm{D} / \mathrm{RPKAD}$ & $\mathrm{J}$ \\
\hline 2. vii. 68 & 38 & Brig. Gen. Leo Lopulisa & $\mathrm{Sl}$ & Ambonese \\
\hline 28. viii. 71 & 19 & *Brig. Gen. Jasir Hadibroto & $\mathrm{D}$ & $\mathrm{J}$ \\
\hline $3 . \mathrm{iv} .73$ & 22 & $\begin{array}{l}* * \text { Brig. Gen. Alex } \\
\text { Prawiraatmadja }\end{array}$ & $\mathbf{S}$ & S \\
\hline $28 . i .75$ & $30 \frac{1}{2}$ & *Brig. Gen. Sukotjo & $\mathrm{D}$ & $\mathrm{J}$ \\
\hline c. 14. viii. 77 & 31 & *Brig. Gen. Ismail & $\mathbf{D}$ & $\mathbf{J}$ \\
\hline 10. iii. 80 & 14 & **Brig. Gen. M. Sanif & $\mathrm{S} l$ & $\mathbf{S}$ \\
\hline $16 . \mathrm{v} .81$ & & Brig. Gen. Edy Sudradjat & Sl & $J$ \\
\hline
\end{tabular}


Kodam III 17 Agustus (West Sumatra)

\begin{tabular}{|c|c|c|c|}
\hline 31.iii. 57 & 12 & Lt. Col. Ahmad Husein & W Sumatra \\
\hline $12 . \mathrm{iv} .58$ & $3 \frac{1}{2}$ & Col. Ahmad Yani & $\mathrm{D}$ \\
\hline 24. vii. 58 & 7 & *Col. Pranoto Reksosamudro & $\mathrm{D}$ \\
\hline 3.iii. 59 & 6 & *Col. Surjosumpeno & $\mathrm{D}$ \\
\hline $10 . i x .59$ & 4 & *Lt. Col. Panudju & $\mathrm{D}$ \\
\hline 5.1 .60 & 46 & $* *$ Brig. Gen. Surjosumpeno & $\mathbf{D}$ \\
\hline $29 . x .63$ & 6 & Col. Soewito Harjoko & $\mathrm{D}$ \\
\hline $20 . i v \cdot 64$ & $21 \frac{1}{2}$ & **Brig. Gen. Panudju & $\mathrm{D}$ \\
\hline 5.ii. 66 & 30 & *Brig. Gen. S. Poniman & $\mathrm{S} 1$ \\
\hline 9. viii. 68 & 20 & *Brig. Gen. Widodo & $\mathrm{D}$ \\
\hline $17 . \mathrm{iv} .70$ & 46 & Brig. Gen. Sumantoro & $\mathrm{D}$ \\
\hline $6 . \mathrm{ii} .74$ & 48 & Brig. Gen. Sutedjo & $\mathrm{D}($ or $\mathrm{B}$ ?) \\
\hline $30 . \dot{i} .78$ & $15 \frac{1}{2}$ & Brig. Gen. Sumaryo Martosaputro & $\mathrm{D}$ \\
\hline 19.v. 79 & 20 & Brig. Gen. Soelarso & $\mathrm{D}$ \\
\hline $26 . \mathrm{i} .81$ & & Brig. Gen. Sarwono & $\mathrm{S} 1 ?$ \\
\hline
\end{tabular}

$T \& T I I$

$T \& T$ II Sriwijaya (South Sumatra/Jambi)

$\begin{array}{lllll}\text { [27.xii.49 } & 3 & * \text { Col. Maludin Simbolon } & \text { N \& S Sum. } & \text { Toba Batak] } \\ \text { 30.iii.50 } & 29 & * \text { Col. Bambang Utojo } & \text { S Sumatra } & \text { J } \\ \text { 5.ix.52 } & 2 \frac{1}{2} & * \text { Lt. Col. R. A. Kosasih [acting] }{ }^{2} & \text { S1 } & \text { S } \\ 25 . x i .52 & 30 \frac{1}{2} & * * \text { Col. Bambang Utojo } & \text { S Sumatra } & \text { J } \\ \text { 11.vi.55 } & 13 & \text { Lt. Col. Dr. Ibnu Sutowo } & \text { S Sumatra } & \text { J } \\ \text { 2.vii.56 } & 24 & \text { Lt. Col. Barlian } & \text { S Sumatra } & \text { S Sumatran } \\ \text { 26. vi.58 } & & \text { Col. Harun Sohar } & \text { S Sumatra } & ?\end{array}$

2. As Acting Commander at the time of the October 17, 1952 affair, Lt. Col. Kosasih was unwilling to take sides in it, and a group headed by Lt. Col. Kretarto seized power from him on November 23, 1952. The central government condemned the action, and reappointed the former panglima, Col. Bambang Utojo, who was acceptable to both sides. On the internal dissension in the army at the time of the October 17 affair, see Herbert Feith, The Decline of Constitutional Democracy in Indonesia (Ithaca: Cornell University Press, 1962), pp. 246-73. 


\section{Kodam IV}

Kodam IV Sriwijaya (South Sumatra and Jambi), inaugurated February 1, 1961

$\begin{array}{llcll} & 50 & \text { Col. Harun Sohar } & \\ \text { 28.viii.62 } & 58 & * \text { Brig. Gen. Makmun Murod } & \text { S Sumatra } & ? \\ \text { 17.vi.67 } & 31 \frac{1}{2} & \text { **Maj. Gen. M. Ishak Djuarsa } & \text { Sl } & \text { S Sumatran } \\ \text { 7.ii.70 } & 22 & \text { Brig. Gen. Satibi Darwis } & \text { Sl } & \text { S } \\ \text { 30.xi.71 } & 41 \frac{1}{2} & \text { *Brig. Gen. R. Himawan Soetanto } & \text { Sl } & \text { S } \\ \text { 9.v.74 } & 21 \frac{1}{\frac{1}{2}} & \text { Maj. Gen. Amir Judowinarno } & \text { D } & \text { J } \\ \text { 26.ii.76 } & 20 & \text { **Brig. Gen. Haroen Soewardi } & \text { B } & \text { J } \\ \text { ?.x.77 } & 19 & \text { Brig. Gen. Obrin Setyakusumah } & \text { Sl } & \text { S } \\ \text { 18.v.79 } & 43 & \text { *Brig. Gen. Try Soetrisno } & \text { B/Zeni } & \text { J } \\ \text { 24.xii.82 } & & \text { Brig. Gen. Arie Bandiyoko } & \text { B/Zeni } & \text { J }\end{array}$

\section{JAVA}

$T \& T I I I$

$T \& T$ III Siliwangi (West Java)

\begin{tabular}{lcrll}
\multicolumn{2}{c}{$15 . x i .51$} & $*$ Col. Sadikin & S1 & J \\
15.xi.51 & 57 & $* * *$ Col. Alex Evart Kawilarang & S1 & Menadonese \\
14.viii.56 & $7 \frac{1}{2}$ & Col. Suprajogi & S1 & J \\
27.iii.57 & & Col. R. A. Kosasih [acting] & Sl & S \\
20.ii.58 & & (**Col. R. A. Kosasih & S1 & S
\end{tabular}

Following the Keputusan KSAD of October 24, 1959 changing Army Territories into Kodams, the former $T$ \& $T$ III was divided into Kodam V Jaya (Jakarta) and Kodam VI Siliwangi (West Java).

\section{Kodam $V$}

Kodam V Jaya (Jakarta)
18.i. $60 \quad 70 \frac{1}{2}$
Maj. Gen. R. Umar Wirahadi-
S1
$\mathbf{S}$
7.xii. $65 \quad 37 \frac{1}{2}$ kusumah
26.i. 69
14
**Maj. Gen. Amir Machmud
SI
$\mathbf{S}$
***Maj. Gen. Makmun Murod
S1
S Sumatran

3. Lt. Col. Soewito Harjoko was acting panglima for several months ending March 10, 1961, while Col. Sohar was absent through illness. 


$\begin{array}{lllll}23 . \text { iii.70 } & 38 & * * * \text { Maj. Gen. S. Poniman } & \text { S1 } & \text { J } \\ \text { 17.iv.73 } & 54 & * * \text { Lt. Gen. G. H. Mantik } & \text { Sl } & \text { Menadonese } \\ \text { 12.x.77 } & 62 & * * \text { Lt. Gen. Norman Sasono } & \text { CPM } & \text { J } \\ \text { 27.xii.82 } & & * * \text { Maj. Gen. Try Soetrisno } & \text { B/Zeni } & \text { J }\end{array}$

\section{Kodam VI}

Kodam VI Siliwangi (West Java)

\begin{tabular}{|c|c|c|c|c|}
\hline & 29 & $* * *$ Col. R. A. Kosasih & S1 & $\mathbf{S}$ \\
\hline 16. viii. 60 & 71 & Maj. Gen. Ibrahim Adjie & Sl & $\mathbf{S}$ \\
\hline 20. vii. 66 & 33 & Maj. Gen. Hartono Rekso Dharsono & SI & $J$ \\
\hline $19 . \mathrm{iv} .69$ & 41 & $\begin{array}{l}* * \text { Maj. Gen. Antonius Johannes } \\
\text { Witono Sarsono }\end{array}$ & SI & $J$ \\
\hline $16 . \mathrm{ix} .72$ & 14 & Maj. Gen. Wahju Hagono & S1 & $J$ \\
\hline $14 . \mathrm{i} .74$ & 12 & **Maj. Gen. Aang Kunaefi Kartawirja & S1 & $\mathbf{S}$ \\
\hline 15.i. 75 & 45 & **Maj. Gen. R. Himawan Soetanto & Sl & $\mathbf{J}$ \\
\hline $21 . x .78$ & 53 & Maj. Gen. R. Yogie Suwardi Memet & Sl/RPKAD & Cirebon? \\
\hline \multicolumn{5}{|c|}{$T \& T I V$} \\
\hline \multicolumn{5}{|c|}{$T \& T$ IV Diponegoro (Central Java) } \\
\hline$? . x i i .50$ & 21 & *Col. Gatot Subroto & $\mathrm{D}$ & $\mathbf{J}$ \\
\hline $20 . \mathrm{ix} .52$ & $47 \frac{1}{2}$ & Col. Moch. Bachroen & $\mathrm{D}$ & $\mathrm{J}$ \\
\hline $3 . i x .56$ & 37 & Col. Soeharto & $\mathrm{D}$ & $J$ \\
\hline
\end{tabular}

On 27.x.59 T\& TIV became Kodam VII/Diponegoro

Kodam VII

Kodam VII Diponegoro (Central Java)

\begin{tabular}{|c|c|c|c|}
\hline $14 . x .59$ & $19 \frac{1}{2}$ & **Col. Pranoto Reksosamudro & D \\
\hline $26 . v \cdot 61$ & $39 \frac{1}{2}$ & $* *$ Brig. Gen. Sarbini & D \\
\hline $10 . \mathrm{ix} .64$ & 21 & ***Maj. Gen. Surjosumpeno & $\mathrm{D}$ \\
\hline $12 . \mathrm{ix} .66$ & 46 & Maj. Gen. Surono ${ }^{4}$ & $\mathrm{D}$ \\
\hline $23 . i v \cdot 70$ & $35 \frac{1}{2}$ & **Maj. Gen. Widodo & $\mathrm{D}$ \\
\hline $7 . \mathrm{iv} .73$ & $52 \frac{1}{2}$ & $* *$ Maj. Gen. Jasir Hadibroto & $\mathrm{D}$ \\
\hline 19. viii. 77 & $6 \frac{1}{2}$ & Maj. Gen. Sumitro & $\mathrm{D}$ \\
\hline
\end{tabular}

4. Surono had been acting panglima since June 18, 1966 . 
4. iii. 78

35

**Maj. Gen. Sukotjo

D J

$27 . \dot{1} .81$

**Maj. Gen. Ismail

D

$\mathrm{J}$

\section{$T \& T V$}

$T \& T V$ Brawijaya (East Java)

$\begin{array}{lclll}-- & 9 . v i .50 & \text { Col. Sungkono } & \text { B } & \text { J } \\ \text { 9.vi.50 } & 28 \frac{1}{2} & \text { Col. Bambang Sugeng } & \text { C Java } & \text { J } \\ 24 . \times .52 & - & \text { Lt. Col. Dr. Suwondho [acting] } & \text { B } & \text { J } \\ 31 . x .52 & & \text { (Lt. Col. R. Sudirman [acting] } & \text { B } & \text { J } \\ \text { 1.viii.54 } & 44 & \text { (Col. R. Sudirman } & \text { B } & \text { J } \\ 11 . v i i .56 & 2 & * \text { Col. Basuki Rachmat [acting] } & \text { B } & \text { J } \\ 12 . i x .56 & 37 \frac{1}{2} & * \text { Col. Sarbini } & \text { D } & \text { J }\end{array}$

Kodam VIII

Kodam VIII Brawijaya (East Java) was inaugurated on October 21, 1959.

$\begin{array}{lllll}21 . x .59 & 35 & \text { Brig. Gen. Surachman } & \text { B } & \text { J }\end{array}$

29.ix.62 ( Brig. Gen. Basuki Rachmat [acting] B J

15.xii.62 $38 \quad$ **Brig. Gen. Basuki Rachmat $\quad$ B $\quad J$

$\begin{array}{lllll}26 . x i .65 & 7 & * * \text { Brig. Gen. R. Sunarijadi } & \text { B } & J\end{array}$

17.vi.66 $10 \quad * *$ Maj. Gen. Sumitro $\quad$ B $\quad J$

15.iv.67 $33 \quad * *$ Maj. Gen. Moch. Jasin $\quad$ B $\quad J$

22.i.70 23 Maj. Gen. Wahono $\quad$ B $J$

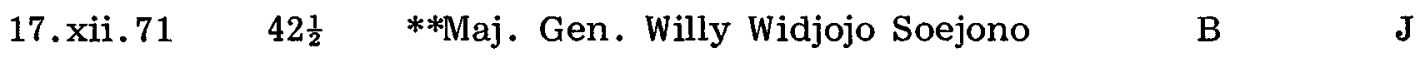

3.vi.75 $71 \quad$ Lt. Gen. Witarmin $\quad$ B $J$

9.v.81 **Maj. Gen. Murgito $\quad$ B

\section{KALIMANTAN}

$T \& T V I$

$T \& T$ VI Tanjungpura (Kalimantan)

$\begin{array}{lllll}8.1 .50 & 22 \frac{3}{2} & \text { Lt. Col. R. Sukanda Bratamenggala } & \text { S1 } & \text { S } \\ 21 . x i .51 & 56 & * * \text { Col. Sadikin } & \text { Sl } & \text { J }\end{array}$


10.viii.56 11 Col. Abimanju C Java J

9.vii.57 12 Col. Kusno Utomo Widjojokerto Sl Banten? J?

On July 17, 1958, T \& T VI was divided into four Regional Military Commands (KDM, later Kodam): Kalimantan Selatan, Kalimantan Timur, Kalimantan Barat, and Kalimantan Tengah, in accordance with the Ministry of Defense Keputusan A/112/58, dated January 31, 1958.

\section{Kodam IX}

Kodam IX Mulawarman (East Kalimantan)

$\begin{array}{lllll}\text { 18.vii.58 } & 22 & \text { Lt. Col. R. Hartojo } & \text { D } & \text { J } \\ \text { 16.v.60 } & 57 & \text { Brig. Gen. R. Soeharjo } & \text { B } & \text { J } \\ \text { 16.ii.65 } & 8 \frac{1}{2} & \text { *Brig. Gen. Sumitro } & \text { B } & \text { J } \\ \text { 5.xi.65 } & 52 & \text { Maj. Gen. Mung Parhadimuljo } & \text { B/RPKAD } & \text { J } \\ \text { 31.iii.70 } & 21 & \text { **Brig. Gen. Sukertijo } & \text { B } & \text { J } \\ \text { c. 29.xii.71 } & 15 & \text { *Brig. Gen. G. H. Mantik } & \text { Sl } & \text { Menadonese } \\ \text { 4.iv.73 } & 22 & \text { Brig. Gen. Sukotjo } & \text { D } & \text { J } \\ \text { 8.ii. } 75 & 40 & \text { Brig. Gen. Ery Supardjan } & \text { D } & \text { J } \\ \text { 6.vi.78 } & 28 & \text { Brig. Gen. Rachwono } & \text { D } & \text { J } \\ 11 . x .80 & 7 & \text { *Brig. Gen. Murgito } & \text { B } & \text { J } \\ 19 . v .81 & & \text { Brig. Gen. Henry Santosa } & \text { D } & \text { J }\end{array}$

Kodam X

Kodam X Lambung Mangkurat (South Kalimantan)

\begin{tabular}{|c|c|c|c|c|}
\hline 17. vii. 58 & 2 & Col. H. Hasan Basry & S Kal & Banjarese \\
\hline $24 . \mathrm{ix} .58$ & $30 \frac{1}{2}$ & Lt. Col. A. Wahab Sjachranie & S Kal & Banjarese \\
\hline $11 . \mathrm{iv} .61$ & $19 \frac{1}{2}$ & Col. M. Jusi & $?$ & $?$ \\
\hline $28 . x i .62$ & $35 \frac{1}{2}$ & *Brig. Gen. Amir Machmud & $\mathrm{S} l$ & $\mathbf{S}$ \\
\hline c. $15 . x i .65$ & 4 & Col. R. Sutopo Juwono [acting] & Sl & $J$ \\
\hline 19. iii. 66 & 40 & **Maj. Gen. Sabirin Mochtar & $\mathrm{B}$ & J? Madurese? \\
\hline 19. vii. 69 & 28 & **Brig. Gen. Hadisoejatno & Zeni & $\mathbf{J}$ \\
\hline $26 . x i .71$ & $26 \frac{1}{2}$ & Brig. Gen. Iksan Soegiarto & $\mathrm{D}$ & $\mathrm{J}$ \\
\hline 14.ii. 74 & 23 & Brig. Gen. Supardjo & $?$ & $\mathrm{~J}$ \\
\hline $5 . \mathrm{i} .76$ & $40 \frac{1}{2}$ & $\begin{array}{l}\text { Brig. Gen. R. Mistar } \\
\text { Tjokrokusumo }\end{array}$ & B & $\mathrm{J}$ \\
\hline
\end{tabular}

5. During the month preceding Abimanju's appointment Lt. Col. Kusno Utomo Widjojokerto was acting panglima. 


$\begin{array}{lllll}\text { 17.v.79 } & 24 & \text { Brig. Gen. Sudiman Saleh } & \text { B/Art } & \text { J } \\ \text { 18.v.81 } & 19 & \begin{array}{l}\text { Brig. Gen. Harjono } \\ \text { Prawirodirdjo }\end{array} & \text { B/Cav } & \text { J } \\ \text { 29.xii.82 } & & \text { Brig. Gen. Syamsuddin } & ? & ?\end{array}$

\section{Kodam XI}

Kodam XI Tambun Bungai (Central Kalimantan)

\begin{tabular}{|c|c|c|c|c|}
\hline 17. vii. 58 & 11 & Lt. Col. Darmo Sugondo & $?$ & $\mathrm{~J} ?$ \\
\hline 18.vi. 59 & c. 7 & Col. Soenitijoso & $\mathrm{D}$ & $J$ \\
\hline c. Feb 60 & 12 & $\begin{array}{l}\text { Lt. Col. Erman Hari- } \\
\text { rustaman [acting] }\end{array}$ & $?$ & $?$ \\
\hline $11 . \mathrm{ii.} 61$ & c. 29 & Col. Darsono Prodjowibowo & $D ?$ & $\mathrm{~J} ?$ \\
\hline $\operatorname{mid} 1963$ & c. $16 ?$ & *Col. P. Sobiran & $\mathrm{B}$ ? & $?$ \\
\hline c. Nov-Dec 64 & c. 16 & *Brig. Gen. Sabirin Mochtar & $\mathrm{B}$ & J? Madurese? \\
\hline 19.iii. 66 & $27 \frac{1}{2}$ & Brig. Gen. Agus Siswadi & $?$ & $\mathrm{~J}$ \\
\hline 6. vii. 68 & $12 \frac{1}{2}$ & *Brig. Gen. Hadisoejatno & Zeni & J \\
\hline 26. vii. 69 & $44 \frac{1}{2}$ & *Brig. Gen. Alex Prawiraatmadja & $\mathbf{S}$ & $\mathbf{S}$ \\
\hline
\end{tabular}

Kodam XI was officially abolished on February 14, 1974, and incorporated into Kodam X. (Brig. Gen. Alex Prawiraatmadja had left ten months previously to become panglima of Kodam II.)

\section{Kodam XII}

The West Kalimantan Command, established July 19, 1958 was officially designated Kodam XII Tanjungpura (West Kalimantan) on February 18, 1960.

\begin{tabular}{|c|c|c|c|c|}
\hline 20. vii. 58 & 17 & Lt. Col. Soeharto & $\mathrm{D}$ & $\mathbf{J}$ \\
\hline $20 . x i i .59$ & 45 & Col. Soedharmo & $?$ & $\mathrm{~J}$ \\
\hline $10 . \mathrm{ix} .63$ & $45 \frac{1}{2}$ & Brig. Gen. Ryacudu & TT II & Lampung \\
\hline 30. vi. 67 & 22 & $\begin{array}{l}\text { *Brig. Gen. Antonius Johannes } \\
\text { Witono Sarsono }\end{array}$ & Sl & $J$ \\
\hline $23 . \mathrm{iv} .69$ & $47 \frac{1}{2}$ & Brig. Gen. Drs. Soemadi & B & $\mathrm{J}$ \\
\hline $11 . \mathrm{iv} .73$ & 29 & Brig. Gen. R. Seno Hartono & $\mathrm{S} 1$ & $\mathbf{J}$ \\
\hline c. $15 . \mathrm{ix} .75$ & 5 & Brig. Gen. R. M. Subandiono & $?$ & $\mathbf{J}$ \\
\hline $20 . \mathrm{ii} .76$ & 20 & *Brig. Gen. Norman Sasono & CPM & $\mathrm{J}$ \\
\hline $8 . x .77$ & 19 & Brig. Gen. Subhan Djajaatmadja & $\mathrm{Sl}$ & $\mathbf{S}$ \\
\hline $18 . v \cdot 79$ & 10 & *Brig. Gen. M. Sanif & Sl & $\mathbf{S}$ \\
\hline 22.iii. 80 & 33 & Brig. Gen. Untung Sridadi & $\mathrm{D}$ & $\mathrm{J}$ \\
\hline 30.xii. 82 & & Brig. Gen. I. B. Sudjana & $?$ & Balinese? \\
\hline
\end{tabular}




\section{EASTERN INDONESIA}

$T \& T V I I$

$T \& T$ VII Wirabuana (Great East)

[ 26.iv.50 Lt. Col. Achmad Junus Dolang Mokoginta Mongondouw]

24.iv.50 $20 \quad * *$ Col. Alex Evart Kawilarang $\quad$ Sl Menadonese

$\begin{array}{lllll}3.1 .52 & 12 & * * \text { Col. Gatot Subroto } & \mathrm{D} & \mathrm{J}\end{array}$

3.i.53 43 Col. Joop Warouw B

1.viii.56 $18 \quad$ Lt. Col. Ventje Sumual $\quad$ C Java Menadonese

After Permesta was proclaimed in June 1957, the Army Chief of Staff attempted to restrict the potential area of dissidence in eastern Indonesia by dividing $\mathrm{T} \& \mathrm{~T}$ VII into four military districts. These were designated as: North and Central Sulawesi, which later became Kodam XIII Merdeka; South and Southeast Sulawesi, which later became Kodam XIV Hasanuddin; Moluccas and West Irian, which later became Kodam XV Pattimura; and Bali and Sunda Kecil, which later became Kodam XVI Raksabuana (and changed its name in 1960 to Udayana). Lt. Col. Somba was appointed first commander of North and Central Sulawesi, but was dishonorably discharged after the PRRI/Permesta ultimatum of February 1958.

\section{Kodam XIII}

Kodam XIII Merdeka (North/Central Sulawesi)

\begin{tabular}{|c|c|c|c|c|}
\hline $28 . \mathrm{ix} .57$ & 5 & Lt. Col. Daniel Julius Somba & $T \& T$ VII & Menadonese \\
\hline 17.ii. 58 & 7 & Lt. Col. Rukmito Hendraningrat & $\mathrm{D} ?$ & $J$ \\
\hline $23 . \mathrm{ix} .58$ & 13 & Lt. Col. Mursjid & $\mathrm{S}$ & Jakarta \\
\hline $14 . x .59$ & 13 & *Lt. Col. Sunarijadi & B & $\mathrm{J}$ \\
\hline $17 . x i .60^{6}$ & 58 & $\begin{array}{l}\text { Brig. Gen. Soenandar } \\
\text { Prijosoedarmo }\end{array}$ & B & $\mathbf{J}$ \\
\hline 22. ii. 66 & 20 & Brig. Gen. Sudarmono & $\mathrm{D} ?$ & $\mathbf{J}$ \\
\hline $28 . x .67$ & 29 & Brig. Gen. Kaharuddin Nasution & S/RPKAD & $\begin{array}{l}\text { Mandailing } \\
\text { Batak }\end{array}$ \\
\hline $23 . \mathrm{iii} .70$ & 21 & *Brig. Gen. Willy Widjojo Soejono & B & $\mathrm{J}$ \\
\hline c. $15 . x i i .71$ & $26 \frac{1}{2}$ & Brig. Gen. Julius Henuhili & $\mathbf{S}$ & Timorese \\
\hline $25 . \mathrm{ii} .74$ & 22 & Brig. Gen. E. W. P. Tambunan & B & Toba Batak \\
\hline $8 . i .76$ & 32 & Brig. Gen. Edy Sugardo & $\mathbf{B}$ & $\mathbf{J}$ \\
\hline $16 . \mathrm{ix} .78$ & $28 \frac{1}{2}$ & Brig. Gen. Rudini & B & $J$ \\
\hline $28 . i .81$ & & $\begin{array}{l}\text { Brig. Gen. Drs. Susanto } \\
\text { Wismoyo }\end{array}$ & Cavalry & $J$ \\
\hline
\end{tabular}

6. At the time of the changeover, Chief of Staff of Kodam XIII Lt. Col. R. Bintoro was Acting Commander. 
Kodam XIV

Established in June 1957, the command on October 24, 1959 was officially named Kodam XIV Hasanuddin (South and Southeast Sulawesi)

$\begin{array}{lllll}\text { 1.vi.57 } & 29 & \text { Lt. Col. Andi Mattalatta } & \text { Hasanuddin } & \text { Buginese } \\ \text { 6.xi.59 } & 73 \frac{1}{2} & \text { Brig. Gen. Andi Moh. Jusuf } & \text { Hasanuddin } & \text { Buginese } \\ \text { 27.xii.65 } & 32 & \text { Brig. Gen. Solihin G.P. } & \mathrm{S} 1 & \mathrm{~S} \\ \text { 3.viii.68 } & 19 \frac{1}{2} & \text { Brig. Gen. Sajidiman } & \mathrm{Sl} & \mathrm{J} \\ \text { 16.iii.70 } & 37 & \text { Brig. Gen. Abdul Azis Bustam } & \text { Hasanuddin } & \text { Buginese } \\ \text { 10.iv.73 } & 29 \frac{1}{2} & \text { Brig. Gen. Hasan Slamet } & \mathrm{S} 1 & \mathrm{~S} \\ \text { 22.ix.75 } & 22 \frac{1}{2} & \text { Brig. Gen. H. Endang Sukma } & \mathrm{S} 1 & \mathrm{~S} \\ \text { 10.viii.77 } & 21 & \text { Brig. Gen. Kusnadi } & \mathrm{D} & \mathrm{J} \\ \text { 19.v.79 } & & \text { Maj. Gen. Soegiarto } & \mathrm{S} 1 & \mathrm{~J}\end{array}$

\section{Kodam XV}

Kodam XV Pattimura covered the Moluccas and West Irian, until a separate command was formed for West Irian (Kodam XVII) in 1962.

\begin{tabular}{|c|c|c|c|c|}
\hline 26. vi. 57 & 42 & Col. Herman Pieters & Ambon & Ambonese \\
\hline $4 . \mathrm{i} .61$ & $61 \frac{1}{2}$ & Brig. Gen. Busjiri & B & $\mathbf{J}$ \\
\hline 15.ii. 66 & $30 \frac{1}{2}$ & Brig. Gen. R. Djohari & $\mathrm{S} 1$ & $\mathbf{S}$ \\
\hline 28. viii. 68 & 19 & $* *$ B rig. Gen. S. Poniman & $\mathrm{Sl}$ & $\mathrm{J}$ \\
\hline 17.iii. 70 & 46 & $\begin{array}{l}\text { Brig. Gen. Wing Wirjawan } \\
\text { Wirjodiprodjo }\end{array}$ & D/Cavalry & $\mathbf{J}$ \\
\hline $19 . i .74$ & $25 \frac{1}{2}$ & *Brig. Gen. Haroen Soewardi & B & $\mathbf{J}$ \\
\hline 2.iii. 76 & 23 & Brig. Gen. Abdul Rahman Suhodo & $\mathrm{D}$ & $\mathrm{J}$ \\
\hline 11.ii. 78 & $38 \frac{1}{2}$ & Brig. Gen. Bagus Sumitro & $\mathbf{B}$ & $\mathbf{J}$ \\
\hline $24 . \mathrm{iv} .81$ & & Brig. Gen. Sebastian Soekoso & RPKAD & $J$ \\
\hline
\end{tabular}

Kodam XVI

Kodam XVI Udayana (Nusatenggara) was first inaugurated as KDM Raksabuana on May 27, 1957.

$\begin{array}{lllll}\text { 5.vii.57 } & 26 & \text { Lt. Col. R. Minggu } & \text { Sl } & \text { J } \\ \text { 12.ix.59 } & 44 & \text { Col. Supardi } & \text { B } & \text { J? } \\ \text { ?.v.63 } & 38 & \text { Brig. Gen. Sjafiudin } & \text { B } & \text { J } \\ \text { c. 12.vii.66 } & 44 \frac{1}{2} & \text { *Brig. Gen. Sukertijo } & \text { B } & \text { J } \\ \text { 26.iii.70 } & 24 & \text { Brig. Gen. R. Suprapto } & \text { D } & \text { J } \\ \text { 25.iii.72 } & 23 & \text { Brig. Gen. Ignatius Jogi Supardi } & \text { Artillery } & \text { J }\end{array}$




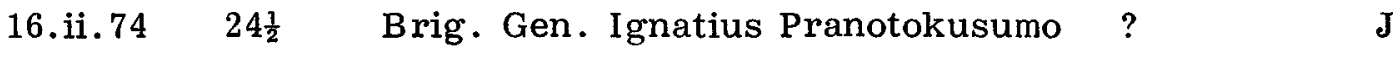

3.iii.76 $31 \quad$ Brig. Gen. Soeweno B/RPKAD J

14.x.78 $53 \quad$ Maj. Gen. Dading Kalbuadi RPKAD J

?.iii.83 Brig. Gen. Sarwono Sl?

\section{Kodam XVII}

Kodam XVII Cendrawasih (Irian Jaya) was formed in August 1962

$\begin{array}{lllll}\text { 8.viii.62 } & 20 & \text { Brig. Gen. U. Rukman } & \text { S1 } & \text { Cirebonese } \\ \text { 17.iv.64 } & 23 & \text { Brig. Gen. R. Kartidjo } & \text { B } & \text { J } \\ \text { 7.iii.66 } & 28 & \text { Brig. Gen. R. Bintoro } & ? & \text { J } \\ \text { 2.vii.68 } & 19 \frac{1}{2} & \text { **Brig. Gen. Sarwo Edhie Wibowo } & \text { D/RPKAD } & \text { J } \\ 20 . i i .70 & 39 \frac{1}{2} & \text { Brig. Gen. Acub Zaenal } & \text { B } & \text { J? Madurese? } \\ 2 . \text { vi.73 } & 19 & \text { Brig. Gen. Kisrad Soetrisno } & \text { D? } & \text { J } \\ 25 . i .75 & 41 \frac{1}{2} & \text { Brig. Gen. Imam Munandar } & \text { B } & \text { J } \\ \text { 8.vii.78 } & 47 & \text { Brig. Gen. Chalimi Imam Santosa } & \text { RPKAD } & \text { J } \\ \text { 14.vi.82 } & & \text { Brig. Gen. R. K. Sembiring } & ? & \text { Karo Batak }\end{array}$


Alphabetical Listing of Territorial Commanders

\author{
Aang Kunaefi Kartawirja \\ Abdulrachman, Djoni \\ Abdulrahman Suhodo \\ Abimanju \\ Acub Zaenal \\ Adjie, Ibrahim \\ Agus Siswadi \\ Amir Judowinarno \\ Amir Machmud \\ Arie Bandiyoko \\ A zis Bustam, Abdul \\ Bachroen, Moch. \\ Bagus Sumitro \\ Bambang Sugeng \\ Bambang Utojo \\ Barlian \\ Basuki Rachmat \\ Bintoro, $R$. \\ Busjiri \\ Dading Kalbuadi \\ Darjatmo \\ Darmo Sugondo \\ Darsono Prodjowibowo \\ Dharsono, H. R. \\ Djohari \\ Endang Sukma, H. \\ Erman Harirustaman \\ Ery Supardjan \\ Gatot Subroto \\ Gintings, Djamin \\ Hadisoejatno \\ Hamzah, Teuku \\ Harahap, A. Rivai \\ Harjono Prawirodirdjo \\ Hartojo, R . \\ Harun Sohar \\ Haroen Soewardi \\ Hasan Basry \\ Hasan Slamet \\ Henuhili, Julius \\ Himawan Soetanto \\ Husein, Ahmad \\ Ibnu Sutowo \\ Iksan Soegiarto \\ Imam Munandar
}

Kodam I 70-73; Kodam VI 74-75

Kodam I 82

Kodam XV 76-78

$T$ \& $T$ VI 56-57

Kodam XVII 70-73

Kodam VI 60-66

Kodam XI 66-68

Kodam IV 74-76

Kodam X 62-65; Kodam V 65-69

Kodam IV 82

Kodam XIV 70-73

T \& T IV 52-56

Kodam XV 78-81

$T \& T$ V 50-52

$T \& T$ II $50-52 ; 52-55$

$\mathrm{T} \& \mathrm{~T}$ II 56-58

$\mathrm{T} \& \mathrm{~T}$ V 56 ; Kodam VIII 62-65

Kodam XVII 66-68

Kodam XV 61-66

Kodam XVI 78-83

Kodam II 63-65

Kodam XI 58-59

Kodam XI 61-63

Kodam VI 66-69

Kodam XV 66-68

Kodam XIV 75-77

Kodam XI 60-61

Kodam IX 75-78

$T$ \& $T$ IV $50-52 ; T$ \& $T$ VII $52-53$

$T$ \& $T$ I 56-58; Kodam II 58-61

Kodam XI 68-69; Kodam X 69-71

Kodam I 67-70

Kodam I 73-78

Kodam X 81-82

Kodam IX 58-60

$T$ \& $T$ II 58 ; Kodam IV 58-62

Kodam XV 74-76; Kodam IV 76-77

Kodam X 58

Kodam XIV 73-75

Kodam XIII 71-74

Kodam IV 71-74; Kodam VI 75-78

Kodam III 57-58

$\mathrm{T} \& \mathrm{~T}$ II $55-56$

Kodam X 71-74

Kodam XVII $75-78$ 


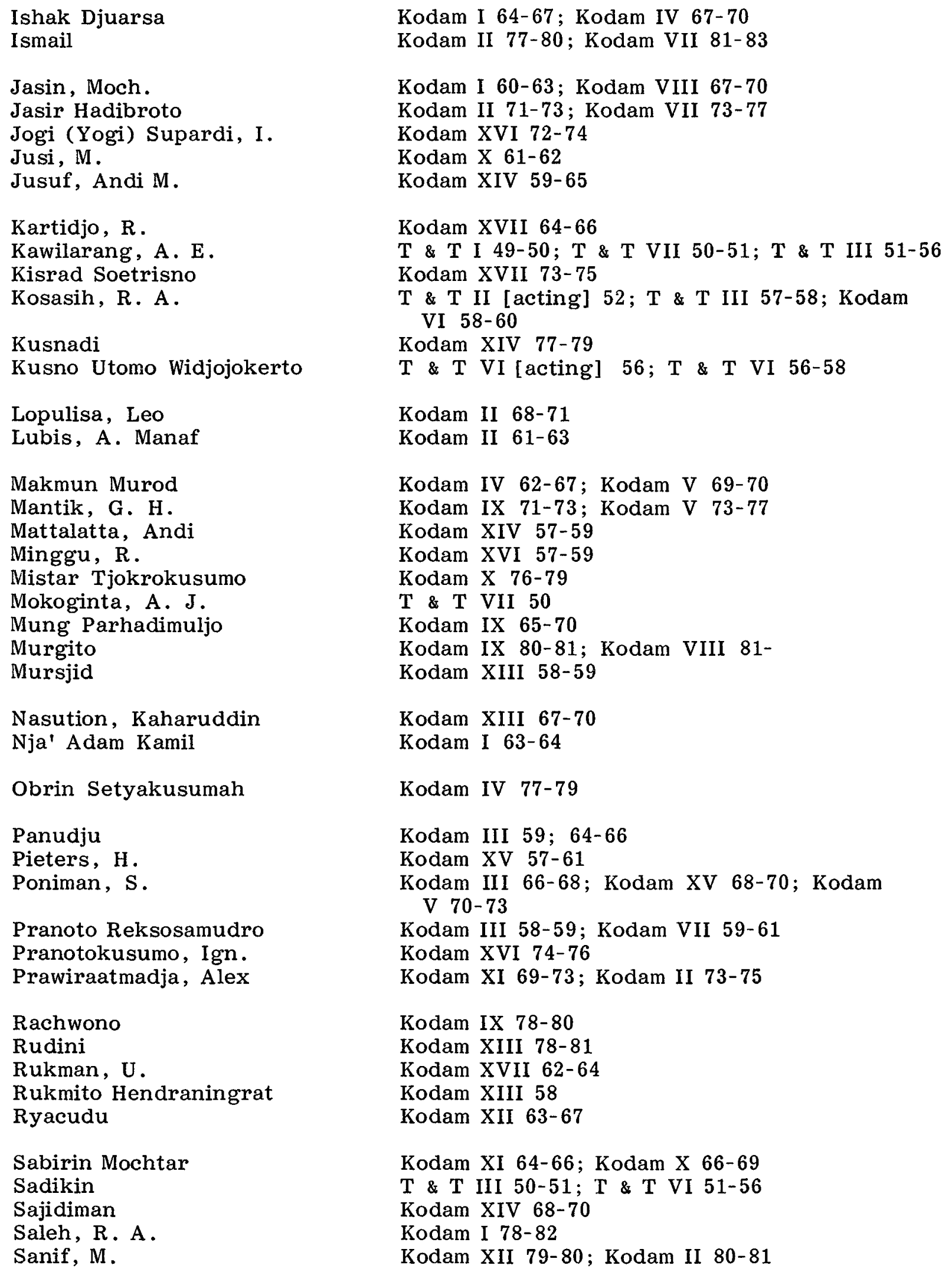




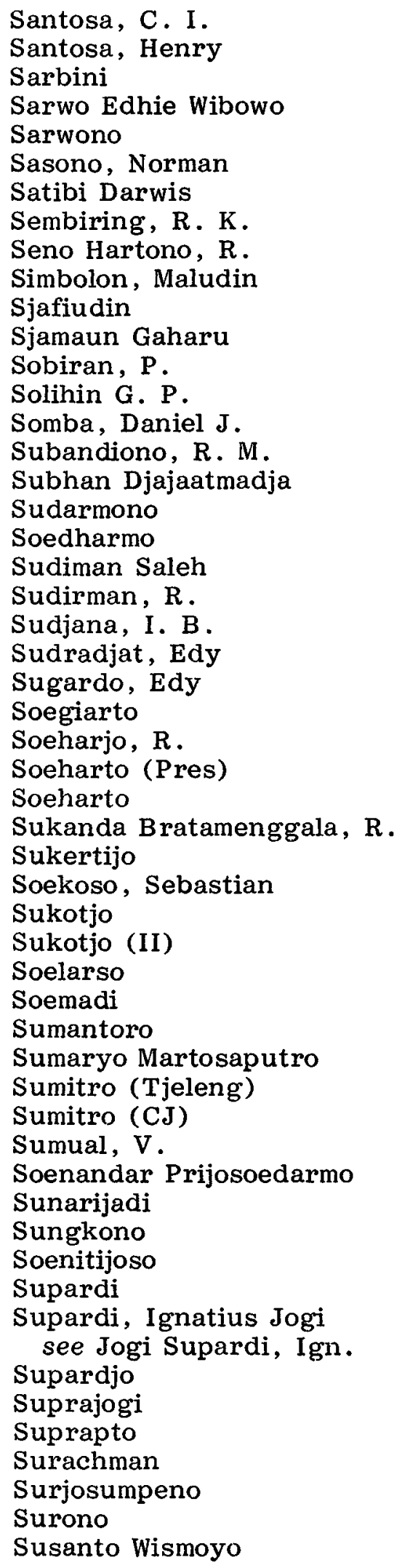

Kodam XVII 78-82

Kodam IX 81-

T \& T V 56-59; Kodam VII 61-64

Kodam II 67-68; Kodam XVII 68-70

Kodam III 81-

Kodam XII 76-77; Kodam V 77-82

Kodam IV 70-71

Kodam XVII 82-

Kodam XII 73-75

$\mathrm{T} \& \mathrm{~T}$ II $49-50 ; \mathrm{T} \& \mathrm{~T}$ I $50-56$

Kodam XVI 63-66

Kodam I 57-60

Kodam XI 63-64; Kodam II 65-67

Kodam XIV 65-68

Kodam XIII 57-58

Kodam XII 75-76

Kodam XII 77-79

Kodam XIII 66-67

Kodam XII 59-63

Kodam X 79-81

$T$ \& $\mathrm{T}$ V 52-56

Kodam XII 82-

Kodam II 81-

Kodam XIII 76-78

Kodam XIV 79-

Kodam IX 60-65

$T \& T$ IV 56-59

Kodam XII 58-59

$T$ \& $T$ VI 50-51

Kodam XVI 66-70; Kodam IX 70-71

Kodam XV 81-

Kodam IX 73-75

Kodam II 75-77; Kodam VII 78-81

Kodam III 79-81

Kodam XII 69-73

Kodam III 70-74

Kodam III 78-79

Kodam IX 65; Kodam VIII 66-67

Kodam VII 77-78

$\mathrm{T} \& \mathrm{~T}$ VII $56-57$

Kodam XIII 60-66

Kodam XIII 59-60; Kodam VIII 65-66

$\mathrm{T} \& \mathrm{~T}$ V 50

Kodam XI 59-60

Kodam XVI 59-63

Kodam X 74-76

$T$ \& $T$ III $56-57$

Kodam XVI 70-72

Kodam VIII 59-62

Kodam III 59; 60-63; Kodam VII 64-66

Kodam VII 66-70

Kodam XIII 81- 
Sutedjo

Sutopo Juwono

Soeweno

Soewito Harjoko

Suwondho

Syamsuddin

Tambunan, E. W. P.

Thalib, A.

Try (Tri) Soetrisno

Umar Wirahadikusumah

Untung Sridadi

Wahab Sjachranie, A.

Wahju Hagono

Wahono

Warouw, Joop

Widjojo Soejono, W.

Widodo

Wing Wirjawan Wirjodiprodjo

Witarmin

Witono Sarsono, A. J.

Yani, Ahmad

Yogie S. Memet, R.
Kodam III 74-78

Kodam X 65-66

Kodam XVI 76-78

Kodam III 63-64; Kodam IV [acting] 60-61

$\mathrm{T} \& \mathrm{~T} \mathrm{~V}$ [acting] 52

Kodam X 82-

Kodam XIII 74-76

Kodam II 63

Kodam IV 79-82; Kodam V 82-

Kodam V 60-65

Kodam XII 80-82

Kodam X 58-61

Kodam VI 72-74

Kodam VIII 70-71

T \& T VII 53-56

Kodam XIII 70-71; Kodam VIII 71-75

Kodam III 68-70; Kodam VII 70-73

Kodam XV 70-74

Kodam VIII 75-81

Kodam XII 67-69; Kodam VI 69-72

Kodam III 58

Kodam VI 78-83 